\title{
Modifications in Near-Surface Layer of Transparent Dielectrics Ionized by Ultrashort Laser Pulses
}

\author{
V. KOMOLOV* AND S. PRZHIBEL'SKII
}

St. Petersburg State University of Information Technologies,

Mechanics and Optics

Kronverkskii pr., 49, St. Petersburg, 197101, Russia

\begin{abstract}
Formation of spatially non-uniform mechanical tension in a transparent solid due to the lattice ionization under the intensive ultrashort laser pulses action is predicted and theoretically described. Within the framework of the continual theory of elasticity the estimation of both average values of deformation tensor and its variations in solid dielectric with non-uniform random distribution of ions was carried out. It was shown that three possible types of medium modification can arise in solid depending on its ionization degree and the thickness of ionized layer. Since the Coulomb fields inside ionized layer can be non-uniform, all three types of structuring can arise in solid simultaneously.
\end{abstract}

PACS numbers: 81.07.--b, 81.16.-c

\section{Introduction}

In this work we continue to study particularities of the short laser pulse action on the transparent solids. Earlier in Refs. $[1,2]$ we had studied the case when the disturbance of charge equilibrium in a solid due to its ionization under intensive light pulse causes the damage. Now we analyze the possible solid response under the action of pulses with "moderate" intensity, when the arising Coulomb forces are too weak to cause damage, but strong enough to create the nanoscale defects in the near-surface layer of solid. The analysis is carried out in the frames of continual model of the medium that allows obtaining the analytical solutions, much more simple and descriptive than can be received by cumbersome numerical methods that take into account the discreteness of examined system. The current letter contains only the brief description of our approach and the main results of analysis, the description of the method and details of solutions will be published in Journal of Optical Technology.

*corresponding author; e-mail: komolov@mail333.com 
Intensive short laser pulses can ionize the dielectric medium, forming an array of randomly distributed ions [1-4]. The Coulomb interaction of ions causes their displacement and mechanical stresses inside the medium. When the Coulomb forces are too weak for causing the direct ion emission [4, 5], the induced mechanical strains can be sufficiently strong to stimulate the stretched modification (the "structuring") of the near-surface layer of solid.

During the surface layer ionization, the state of a solid lattice traces the changes of the Coulomb forces arising due to the ionization process. If the laser pulse is too weak to stimulate damage it is possible to "accumulate" the medium ionization by repeated pulses action. Even at small ionization degrees of the medium the thick ionized layer can be formed, and the Coulomb forces will be sufficiently strong at its periphery. Really, for a layer with ionization degree $p$ and thickness $L$ the electric field strength will be $E=4 \pi e p n L$. Fast electrons $(\approx 10 \mathrm{keV})$ created by the ionization of lattice sites, easily pass the distances $\approx 1 \mu \mathrm{m}$ inside the solid, and the $\mu \mathrm{m}$-size layer with $p \approx 10^{-6}-10^{-5}$ can be formed. The Coulomb field strength on the solid surface can reach the value of about $10^{6}-10^{7} \mathrm{~V} / \mathrm{cm}$ that is comparable with the strengths of intramolecular fields.

\section{Results and discussion}

The structuring in the near-surface layer of solid due to redistribution of deformations is substantially defined by ions density $c$ and its distribution inside the layer. A charge, regularly distributed in the layer, may cause only its homogeneous expansion that does not lead to medium structuring. Actually the charge is always distributed non-uniformly due to its discreteness and fluctuations of the separate ions positions. These fluctuations can originate from the structures formed inside the solid by the local lattice strains. The Coulomb fields action on the separate ions causes its displacement and creates non-local deformations. The size of such deformed areas depends on the local value of the Coulomb forces and is different at the various depths across the layer.

When the Coulomb forces are greater than intramolecular ones, the ions can be displaced considerably, so that the deformed areas of a lattice arise at the scales that noticeably exceed a cell size, but remain less than the average distances between neighbor ions. In this process relatively large strained areas can be formed inside the solid.

The charge distribution can be evaluated using the models that take into account both electron removal from solid and its capture to the traps but this estimation are far outside from the subject-matter of this paper. The obtained results depend strongly on the chosen model.

In the present study we use the simplest model that gives an exponential distribution of the ionization degree across the surface area. We use also the continual approach to estimate the stresses which arise under the Coulomb forces action inside the ionized layer. 
We assume that the identical charges $q$ are distributed in semi-infinite medium $(z>0)$. The Coulomb force acting on each of them is

$$
\boldsymbol{F}_{a}=q^{2} \sum_{b}\left(\boldsymbol{x}_{a}-\boldsymbol{x}_{b}\right) /\left|\boldsymbol{x}_{a}-\boldsymbol{x}_{b}\right|^{3}
$$

The force $\boldsymbol{F}_{a}$ acting on site $a$ induces the medium deformation; at the distance $\boldsymbol{x}$ from the site it can be characterized by the deformation vector [6]:

$$
\boldsymbol{u}_{a}(\boldsymbol{x})=\frac{1+\sigma}{8 \pi E(1-\sigma)} \quad \frac{(3-4 \sigma) \boldsymbol{F}_{a}+\boldsymbol{n}_{a}\left(\boldsymbol{n}_{a} \boldsymbol{F}_{a}\right)}{\left|\boldsymbol{x}-\boldsymbol{x}_{a}\right|}
$$

where $\boldsymbol{n}_{a}=\left(\boldsymbol{x}-\boldsymbol{x}_{a}\right) /\left|\boldsymbol{x}-\boldsymbol{x}_{a}\right|, E-$ Young's modulus and $\sigma-$ Poisson's coefficient. The usual value of $\sigma$ for non-elastic media is about $1 / 4-1 / 3$.

Equation (2) is valid at the semi-infinite medium for the charges arising sufficiently deep under the surface. We assume that the relative ion displacements are smaller than intra-atomic distance and hence much smaller than the distances between neighbor ions, so the main part of the ions lies deep enough under the surface and can be studied in terms of infinite medium.

In the point $\boldsymbol{x}$ the total vector of displacement is defined by the sum $\boldsymbol{u}(\boldsymbol{x})=$ $\sum_{a} \boldsymbol{u}_{a}(\boldsymbol{x})$, where the separate terms are the random functions. The displacement and tension distribution inside the medium can be described by the average values $\langle\boldsymbol{u}(\boldsymbol{x})\rangle$ and $\left\langle u_{j}(\boldsymbol{x}) u_{l}(\boldsymbol{y})\right\rangle$. The averaging made for the random disposition of $N$ charges distributed uniformly along the layers $z=$ const and non-uniformly across the layers

$$
\langle\ldots\rangle=\int_{z>0} \ldots \prod_{a} g\left(\boldsymbol{r}_{a}\right) f\left(z_{a}\right) \mathrm{d} z_{a} \mathrm{~d} \boldsymbol{r}_{a}
$$

where

$$
\int_{0}^{\infty} \mathrm{d} z f(z)=1 \text { and } \int_{S} \mathrm{~d} \boldsymbol{r} g(\boldsymbol{r})=1 .
$$

When the ionized layer thickness $L$ is much greater than the distance between ions $c^{-1 / 3}$ ( $c$ is ion density), the averaging procedure can be carried out in two steps. In the thick layers $\left(L c^{1 / 3} \gg 1\right)$ the collective Coulomb forces are much larger than the forces induced by the nearest neighbors, so the force fluctuations are negligibly small. As the average distribution of ions is identical in any plane parallel to the surface, the Coulomb forces are directed normally to the surface in the main part of layer volume. Therefore, the normal component of this force $F(z)$ is defined by the equation

$$
F(z)^{\prime}=4 q^{2} c(z)=4 \pi q^{2} c_{0} f(z) .
$$

The function $f(z)=\exp (-z / L) / L$ is introduced to account the possible capture of electrons that move towards the surface. The final expression for $F(z)$ may be written as $F(z)=2 \pi q^{2} c_{0} L[1-2 \exp (-z / L)]$ for $z>0$. Let us note that $F=0$ at $z=L \ln 2$.

For $g(r)=1 / S$, where $S=2 \pi R^{2}$ is the irradiated area, and $\sigma=1 / 4$, the averaging of displacement 


$$
\boldsymbol{u}(\boldsymbol{x})=\frac{1}{8 \pi E} \sum_{a} \frac{F\left(z_{a}\right)\left[2 \boldsymbol{n}+\boldsymbol{n}_{a}\left(\boldsymbol{n}_{a} \boldsymbol{n}\right)\right]}{\left|\boldsymbol{x}-\boldsymbol{x}_{a}\right|}
$$

of the medium at point $x$ along $z$ gives

$$
\bar{u}_{z}(x)=\langle\boldsymbol{n u}(\boldsymbol{x})\rangle=\frac{N}{8 \pi E} \int \mathrm{d} \boldsymbol{x}_{a} g\left(\boldsymbol{r}_{a}\right) f\left(z_{a}\right) \frac{F\left(z_{a}\right)\left[2+\left(\boldsymbol{n}_{a} \boldsymbol{n}\right)^{2}\right]}{\left|\boldsymbol{x}-\boldsymbol{x}_{a}\right|} .
$$

Here $\boldsymbol{n}$ is the vector, normal to the surface. In asymptotic limit $R \gg L$ for $r \ll R$ Eq. (5) reduces to

$$
\bar{u}_{z}(x)=\frac{7 q^{2} c_{0} L N}{24 \pi E R}[\exp (-z / L)-\exp (-2 z / L)] .
$$

The strain tensor $u_{j l}=\left(\partial_{j} u_{l}+\partial_{l} u_{j}\right) / 2$ has the only non-zero component

$$
u_{z z}=\frac{7 q^{2} c_{0}^{2} L R}{12 E}[2 \exp (-2 z / L)-\exp (-z / L)] \text {. }
$$

For $E=3 \times 10^{12} \mathrm{erg} / \mathrm{cm}^{3}, q^{2}=25 \times 10^{-20} \mathrm{erg} / \mathrm{cm}$, it gives $u_{z z} \sim$ $3 \times 10^{-32} c_{0}^{2} L R$. The critical value $u_{z z} \approx 0.1$ defines the criterion $c_{0}^{2} L R<$ $3 \times 10^{30} \mathrm{~cm}^{-4}$, when the medium ionization does not lead to its damage.

In the vicinity of the selected ion $b$ the medium displacement differs strongly from its average value. The exact value of it may be written as

$$
\begin{aligned}
\boldsymbol{u}(\boldsymbol{x}) & =\frac{1}{8 \pi E} \sum_{a} \frac{F\left(z_{a}\right)\left[2 \boldsymbol{n}+\boldsymbol{n}_{a}\left(\boldsymbol{n}_{a} \boldsymbol{n}\right)\right]}{\left|\boldsymbol{x}-\boldsymbol{x}_{a}\right|} \\
& =\frac{1}{8 \pi E} \frac{F\left(z_{b}\right)\left[2 \boldsymbol{n}+\boldsymbol{n}_{b}\left(\boldsymbol{n}_{b} \boldsymbol{n}\right)\right]}{\left|\boldsymbol{x}-\boldsymbol{x}_{b}\right|}+\frac{1}{8 \pi E} \sum_{a(\neq b)} \frac{F\left(z_{a}\right)\left[2 \boldsymbol{n}+\boldsymbol{n}_{a}\left(\boldsymbol{n}_{a} \boldsymbol{n}\right)\right]}{\left|\boldsymbol{x}-\boldsymbol{x}_{a}\right|},
\end{aligned}
$$

where the second term becomes dominant far from the ion and is close to the average value. Therefore, the displacement nearby the ion $b$ is defined by

$$
\begin{aligned}
\boldsymbol{u}(\boldsymbol{x}) & =\frac{q^{2} c_{0} L}{4 E} \frac{\left[1-2 \exp \left(-z_{b} / L\right)\right]\left[2 \boldsymbol{n}+\boldsymbol{n}_{b}\left(\boldsymbol{n}_{b} \boldsymbol{n}\right)\right]}{\left|\boldsymbol{x}-\boldsymbol{x}_{b}\right|} \\
& +\frac{7 \boldsymbol{n} q^{2} c_{0} L N}{24 \pi E R}\left[\exp \left(-z_{b} / L\right)-\exp \left(-2 z_{b} / L\right)\right],
\end{aligned}
$$

where the individual contribution of the ion to longitudinal displacement is

$$
u_{z}^{*}(\boldsymbol{x})=\frac{q^{2} c_{0} L\left[1-2 \exp \left(-z_{b} / L\right)\right]\left(2+\cos ^{2} \theta\right)}{4 E|\boldsymbol{x}|} .
$$

The above expressions allow to estimate both the tensor value $u_{z z}^{*}$ and the size of the area nearby the ion where its contribution to $u_{z z}$ dominates. As the Coulomb force depends on the ion position $z_{b}$ the average and peculiar contributions depend variously on $z$ (Fig. 1). For the other tensor components the peculiar contribution is always dominant. It may be shown that the peculiar contribution exceeds an average one inside the area that exceeds the size of a lattice site $\left(\approx 3 \times 10^{-8} \mathrm{~cm}\right)$ when $c_{0} R<10^{15} \mathrm{~cm}^{2}$. 


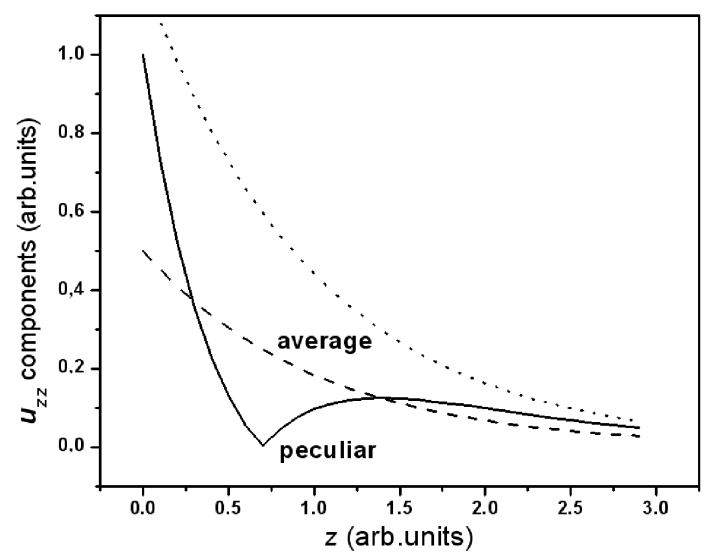

Fig. 1. Average and peculiar contributions to the strain tensor across ionized layer.

\section{Conclusions}

In the framework of a continual theory of elasticity it has been shown that ionization of a transparent solid by the action of non-destructive ultrashort pulses can produce the nanosize areas of the mechanical strains in the near-surface layer of dielectric. The main characteristics of the arising strain field are defined by the ionization degree, the size and configuration of the ionized area. It was assumed in our study that ions density decreases exponentially from the surface to the bulk of the layer but generally the ions space distribution can be controlled by selection of the proper distribution of laser field.

It has been shown that three types of structuring are possible. The first type can be realized when the Coulomb forces acting on the individual ion are small in comparison with intramolecular ones. Then the local non-interacting lattice defects having the sizes about the size of a lattice cell can be created. The second type of structuring arises when the Coulomb forces are greater than intramolecular ones. In this case the ions can be displaced appreciably, so that the deformed areas of a lattice arise at the scales that noticeably exceed a cell size, but remain smaller than the average distances between neighbor ions. In this process relatively large (but not overlapping) strained areas can be formed inside the solid. And, at last, the third type of structuring arises when the displacements of ions are so large that the "strained" areas in the solid are strongly overlapping. In this case almost homogeneous expansion of a lattice takes place.

Since the Coulomb fields inside ionized layer can be non-uniform, all three types of structuring can arise in solid simultaneously.

\section{Acknowledgments}

The work was partly supported by the Russian Foundation for Basic Research (grant 05-02-17534) and the Program of Support for Scientific Schools (grant 7952.2006.2). 


\section{References}

[1] V.E. Gruzdev, V.L. Komolov, S.G. Przhibel'skii, D.S. Smirnov, Proc. SPIE 6256, $30(2006)$.

[2] V.E. Gruzdev,V.L. Komolov, S.G. Przhibel'skii, D.S. Smirnov, J. Opt. Technol. 73, 378 (2006).

[3] D. Ashkenasi, H. Varel, A. Rosenfeld, F. Noack, E.E.B. Campbell, Appl. Phys. A 63, 103 (1996).

[4] W. Kautek, J. Krueger, M. Lenzner, S. Sartania, Ch. Spielmann, F. Krausz, Appl. Phys. Lett. 69, 3146 (1996).

[5] C. Jungreuthmayer, M. Geissler, J. Zanghellini, T. Brabec, Phys. Rev. Lett. 92, 133401 (2004).

[6] L.D. Landau, E.M. Lifshits, Theory of Elasticity, Nauka, Moscow 1965. 